\title{
New Insights into Mechanisms of Oxyradical and Neutrophil Mediated Lung Injury
}

\author{
P.A. Ward and M.S. Mulligan \\ Department of Pathology, The University of Michigan Medical School, Ann Arbor
}

\begin{abstract}
Summary. Acute lung injury in rats developing after systemic complement activation or deposition of IgG immune complexes is complement-dependent and oxygen radical-mediated. Recent findings have shown that a soluble complement receptor (sCR1) is capable of attenuating injury. Additional studies have also demonstrated requirements for the cytokines, $\mathrm{TNF} \alpha$, and for L-arginine derived products in lung injury that follows deposition of IgG immune complexes.
\end{abstract}

Key words: Complement $-\mathrm{sCR} 1-\mathrm{TNF} \alpha-\mathrm{L}$-arginine - Oxygen-radicals

Several recent experimental approaches have provided new insights into an understanding of the role of mediators in complement- and neutrophildependent models of acute lung injury. Two models in which toxic oxygen products of neutrophils can be linked to microvascular endothelial cell injury have been employed. In the first, intravenous infusion of purified cobra venom factor (CVF) leads to acute focal destruction of endothelial cells in the pulmonary interstitial microvasculature [1]. The injury occurring in this model is catalase-sensitive and can also be inhibited by the use of the iron chelator, desferroxamine, and hydroxyl radical ( $\left.\mathrm{HO}^{\circ}\right)$ scavengers such as dimethylsulfoxide or dimethylthiourea. The second model of acute lung injury is induced by intraalveolar deposition of

Abbreviations: $\mathrm{BAL}=$ bronchoalveolar lavage; $\mathrm{BSA}=$ bovine serum albumin $; \mathrm{CVF}=$ cobra venom factor $; \mathrm{ELAM}-1=$ endothelial leukocyte adhesion molecule $1 ;$ sCR $1=$ human soluble complement receptor $1 ; \cdot \mathrm{OH}=$ hydroxyl radical; $\mathrm{IL}-1=$ interleukin-1; $\cdot \mathrm{NO}=$ nitric oxide; $\mathrm{NMA}=\mathrm{N}^{\mathrm{G}}$-monomethylarginine; $\mathrm{ONOO}^{-}=$peroxynitrite anion; $\mathrm{O}_{2}^{-}=$superoxide anion; $\mathrm{SOD}=$ superoxide dismutase $\mathrm{TNF} \alpha=$ tumor necrosis factor alpha
IgG immune complexes, produced by airway instillation of antibody to bovine serum albumin (antiBSA) and intravenous injection of BSA [2]. Injury occurring in this model can be inhibited by the same agents that protect against CVF-induced lung injury. The outstanding difference between the two models is the extensive extravascular (interstitial and intraalveolar) accumulation of neutrophils, whereas in the CVF models of lung injury very few neutrophils appear beyond the confines of the vascular compartment.

\section{Protective Effects of sCR1}

While both models of lung injury have been shown to be complement-dependent (based on the ability of multiple intraperitoneal injections of small amounts of CVF to deplete the complement system), availability of the recombinant, glycosylated form of the soluble complement receptor-1 (sCR1) has provided direct evidence for the critical role of complement activation in the induction of lung injury. SCR1 which has an estimated molecular weight of $205 \mathrm{kD}$, is a genetically engineered molecule which ordinarily exits on the external surfaces of cell membranes. It is the natural receptor for $\mathrm{C} 3 \mathrm{~b}$ and $\mathrm{C} 5 \mathrm{~b}$. In the soluble form, sCR1 binds with these molecules in free solution and effectively blocks the action of the $\mathrm{C} 3$ and the $\mathrm{C} 5$ convertases. When tested in vitro, sCR1 in the $\mathrm{pM}-\mathrm{nM}$ range very effectively inhibits activation of complement (as measured by hemolysis of sensitized red cells, formation of $\mathrm{C} 3$ and $\mathrm{C} 5$ anaphylatoxins, and generation of the membrane attack complex, C5b-9). Recently it has been shown that infusion of sCR1 in vivo substantially reduces ischemic myocardial injury in rats after coronary artery occlusion [3]. As well, injury resulting in the reversed passive dermal Arthus reaction in rats is attenuated by treatment with sCR1 [4]. This protection is associated

Manuscripts published in this issue were the matter of a Symposium held at the University of Ulm, April 24-27, 1991 
with diminished accumulation of neutrophils in the dermis.

The intravenous injection of $\mathrm{SCR} 1$ is profoundly protective in the model of CVF-induced lung injury. Depending on the dose of sCR1 employed (1-25 $\mathrm{mg} / \mathrm{kg}$ body weight), protection from injury (as measured by ${ }^{125} \mathrm{I}$-BSA or extravasation of ${ }^{51} \mathrm{Cr}-\mathrm{RBC}$ ) by as much as $90 \%$ can be achieved. ${ }^{1}$ In the case of IgG immune complex induced injury in rat lung, sCR1 is also a potent protective agent. In a dose-dependent manner, as much as $85 \%$ protection from lung injury can be achieved with sCR1. In this case, the protective effects of sCR1 are correlated with diminished accumulation of neutrophils in the lung tissue, as assessed by extraction of myeloperoxidase. These data indicate the critical requirement for complement activation in these models of acute lung injury. In the case of immune complex-induced injury, the data also indicate that accumulation of neutrophils is closely linked to the complement activation process.

Accordingly, sCR1 is a potent inhibitor for complement-dependent inflammatory reactions. One drawback of the human recombinant preparation of sCR1 is its relatively abbreviated half-life in the circulation, being approximately $15 \mathrm{~min}$ in rat plasma. Without glycosylation, the half-life value is even shorter $(1-5 \mathrm{~min})$. It is possible that chemical modification of SCR 1 , such as derivitization with polyethylene glycol, may result in a compound with a greatly prolonged half-life, thereby greatly reducing the amounts needed in humans.

\section{Requirement for TNF $\alpha$ in Acute Lung Injury}

Although IgG immune complex-induced injury of rat lung is complement and neutrophil-dependent and the lung injury is associated with an extensive accumulation in lung of neutrophils, there is compelling evidence that macrophage-generated cytokines play an important role in this model of lung injury. In vitro incubation of rat alveolar macrophages leads to the generation of $\mathrm{O}_{2}^{-}$as well as the synthesis and release of IL- $1 \beta$ and TNF $\alpha[5$, $6,7]$. Substantial levels of these cytokines can be found in bronchoalveolar (BAL) fluids of rats undergoing intrapulmonary deposition of immune complexes $[6,7]$. By the use of a polyclonal goat antibody to murine $\mathrm{TNF} \alpha$ demonstrating crossreactivity to rat $\mathrm{RNF} \alpha$, rats have been injected

\footnotetext{
1 Mulligan MS, Yeh G, Rudolph A, Ward PA (submitted for publication)
}

intravenously with anti-TNF $\alpha$. Such treatment prevents the appearance in BAL fluids of TNF $\alpha$ activity following deposition of IgG immune complexes in rat lung. Under these circumstances, there is profound protection against immune complexinduced lung injury. As measured by intrapulmonary leakage from the vasculature of ${ }^{125} \mathrm{I}$-BSA, treatment with anti-TNF $\alpha$ reduces injury by nearly $90 \%$. The protection associated with this interventional approach has been found to be related to a substantial reduction (circa 66\%) in intrapulmonary accumulation of neutrophils, as defined by extraction from lung tissue of myeloperoxidase [6].

The current hypothesis regarding the requirement of TNF $\alpha$ in IgG immune complex-induced lung injury is considered to be related to the ability of TNF $\alpha$ to cause upregulation of adhesion molecules within endothelial cells. We have recently shown that in vitro incubation of $\mathrm{TNF} \alpha$ with rat pulmonary artery endothelial cells causes upregulation of ELAM-1, which promotes adhesive interactions between endothelial cells and neutrophils. It is assumed that a similar reaction occurs in vivo:

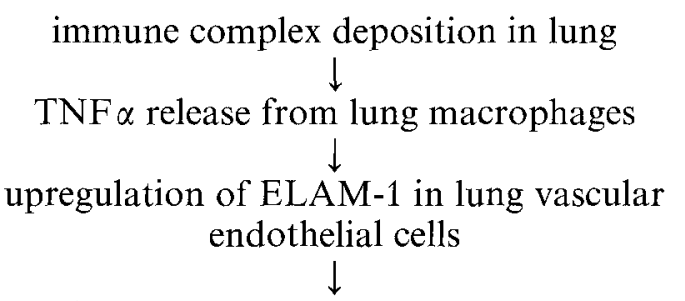

increased adhesive interactions (via ELAM-1) with neutrophils

ability of adherent neutrophils to respond to C5a neutrophil influx into the alveolar compartment

Under this premise, $\mathrm{C} 5 \mathrm{a}$ is unable to function as a chemoattractant in the absence of endothelial cells that have been stimulated to express adhesion molecules. Consequently, the protective effects in vivo of anti TNF $\alpha$ can be explained by the blocking of expression of ELAM-1 (and perhaps other adhesion molecules) on endothelial cells. Experiments are currently underway to assess the validity of this hypothesis.

\section{Requirement for Arginine in Acute Lung Injury}

It is known that L-arginine is required for the cytotoxic properties of activated macrophages on tumor cells [8]. Generation of nitric oxide (NO), a nitrogen centered radical, has been related to 
the cytotoxic process, the target being iron-sulfurcentered enzymes of the mitochondrial electron transport system. "NO is also the chemical structure of the endothelial-derived relaxing factor (EDRF), which is produced by endothelial cells stimulated with acetyl choline. Generation of EDRF leads to relaxation of adjacent smooth muscle cells via stimulation of adenylate cyclase (which results in production of cGMP, the muscle relaxing factor). It is also known that ${ }^{~} \mathrm{NO}$ can interact with $\mathrm{O}_{2}$ - to form the peroxynitrite anion $\left(\mathrm{ONOO}^{-}\right)$ and, ultimately, $\mathrm{HO}^{\circ}$. By-products of this reaction include nitrite $\left(\mathrm{NO}_{2}\right)$ and nitrate $\left(\mathrm{NO}_{3}-\right)$. Potential toxic agents in the arginine pathway would include ' $\mathrm{NO}, \mathrm{ONOO}^{-}, \mathrm{ONOOH}$, and $\mathrm{HO}^{\circ}$. The fact that superoxide dismutase (SOD) is protective against $\mathrm{IgG}$ immune complex-induced lung injury [9] suggests that $\mathrm{O}_{2}{ }^{-}$interaction with $\mathrm{NO}$ may be required for generation of the toxic species. If true, this would suggest that ${ }^{\circ} \mathrm{NO}$ is not the toxic species responsible for tissue injury.

When the arginine analogue, N-monomethylarginine (NMA), is mixed with the anti-BSA, the subsequent lung injury following deposition of IgG immune complexes is greatly attenuated [10]. This protection is reversed by the copresence of L-arginine with NMA but not by the addition of Darginine. Since it is known that D-arginine is not a substrate for ${ }^{\mathrm{NO}}$ synthase and that L-arginine is an absolute requirement and that NMA can function as a competitive analogue for L-arginine, the in vivo data provide compelling evidence for the requirement of $\mathrm{L}$-arginine in the full expression of lung injury following deposition of IgG immune complexes. It seems highly likely that a derivative of ${ }^{\circ O}$ is related to the induction of tissue injury. An interesting feature of the protective effects of NMA in immune complex-induced acute lung injury is the fact that accumulation of neutrophils in lung tissue is not affected by the presence of NMA, in striking contrast to the protective effects of antiTNF $\alpha$ and sCR1 (see above). The ability of NMA to protect the lung from injury following deposition of immune complexes can be monitored by chemical analysis of BAL fluid for the presence of ${ }^{\circ} \mathrm{NO}_{2}$ and ${ }^{\circ} \mathrm{NO}_{3-}$. The presence of $5 \mathrm{mM}$ NMA causes a reduction of these chemicals in BAL fluids by approximately $70 \%$. Thus, there is developing evidence for the role of arginine products in the reduction of acute lung injury associated with complement and neutrophil-dependence acute lung injury. It is likely that this can be linked to generation of "NO although, as stated above, the precise role for the product(s) of-arginine cannot be determined at this time.

\section{Conclusions}

Acute lung injury related to complement activation and neutrophil accumulation within lung tissue involves a complex series of pathophysiological steps and, in some cases, involves the interplay of a network of cells, from which come key intermediate products. This is best seen in the case of $\mathrm{IgG}$ immune complex-induced lung injury where the cytokine TNF $\alpha$ plays a key role in altering the pulmonary microvasculature for the transmigrational responses of neutrophils. These reactions that lead to lung injury also demonstrate an unexpected requirement for-arginine. On the basis of this information, it is now possible to employ new protective interventions that may ultimately have application in human inflammatory diseases.

\section{References}

1. Till GO, Johnson KJ, Kunkel RG, Ward PA (1982) Intravascular activation of complement and acute lung injury. Dependency on neutrophils and toxic oxygen metabolites. J Clin Invest 69:1126-1132

2. Johnson KJ, Ward PA (1974) Acute immunologic pulmonary alveolitis. J Clin Invest 54:349-357

3. Weisman HF, Bartow T, Leppo MK, Marsh HC Jr, Carson GR, Concino MF, Boyle MP, Roux KH, Weisfelot ML, Fearow DT (1990) Soluble complement receptor type 1: in vivo inhibitor of complement supressing post-ischemic myocardial inflammation and necrosis. Science 249:146151

4. Yeh GC, Marsh HC Jr, Carson GR, Berman L, Concino MF, Scesney SM, Kuestner RE, Skibbens R, Donahue KS, Ip SH (1991) Recombinant soluble human complement receptor type $1 \mathrm{sCR} 1$ inhibits inflammation in the reversed passive Arthus reaction in rats. J Immunol 146:250-256

5. Hagenlocker BE, Walker BAM, Ward PA (1990) Superoxide response of immune complex-stimulated rat alveolar macrophages. J Immunol 144:3898-3906

6. Warren JS, Yabroff KR, Remick DG, Kunkel SL, Chensue SW, Kunkel RG, Johnson KJ, Ward PA (1989) Tumor necrosis factor participation in the pathogenesis of acute immune complex alveolitis in the rat. J Clin Invest $84: 1873$ 1882

7. Warren JS (1991) Intrapulmonary interleukin-1 mediates acute immune complex alveolitis in the rat. Biochem Biophys Res Commun 175:604 610

8. Hibbs JB Jr, Trantor RR, Vavrin Z, Rachlin EM (1988) Nitric oxide: a cytotoxic activated macrophage effector molecule. Biochem Biophys Res Commun 157:87-94

9. Johnson KJ, Ward PA (1981) Role of oxygen metabolites in immune complex injury of lung. J Immunol 126:23552359

10. Mulligan MS, Hevel JM, Marletta MA, Ward PA (1991) Tissue injury caused by deposition of immune complexes is L-arginine dependent. Proc Natl Acad Sci USA 88: 6338-6342

Peter A. Ward, M.D

Department of Pathology

The University of Michigan

Medical School

Ann Arbor, MI 48109, USA 